\title{
A Low-Cost Test Approach for Embedded RF Passive Circuits
}

\author{
Abhilash Goyal, Madhavan Swaminathan \\ Georgia Institute of Technology, Department of Electrical and Computer Engineering, Atlanta, USA
}

\begin{abstract}
A new low-cost test approach is proposed for testing embedded RF passive filters (ERPFs) by one-port measurement. By this method, ERPFs testing is possible without a vector network analyzer. This method also enables testing of ERPFs without external test stimulus. In the proposed test approach, a shift in the oscillation frequency of the test-setup is used to detect faults in the filters, but this test approach does not require reconfiguration or conversion of filters into an oscillator as it is done in conventional oscillation-based methods. The core principle of the method is to include an ERPF through a one-port substrate surface probe into an external RF oscillator circuitry, located on the probe card. Such one-port probing causes a change in the oscillation frequency of the oscillator because of the loading from the RF filter, thus enabling low-cost testing of RF filters.
\end{abstract}

\section{INTRODUCTION}

The last decade has seen the use of embedded passives instead of surface mount devices (SMD) in RF front end. This is possible because of the emergence of system of package (SOP) technology [1], which enables reduction in the area of the RF front end [1-3]. SOP technology reduces size of RF front end by embedding RF passive filters in RF substrate, but at the same time it increases the challenges to test these embedded circuits. As shown in Fig. 1, unlike in SMD technology, in the embedded passive technology, only the input and the output ports of the RF passive filter are accessible while internal nodes are not accessible. Inaccessibility of internal nodes increases the challenges to test these embedded RF circuits.

The functional performance of embedded RF passive filters (ERPFs) shifts because of the variations in the fabrication process. The shift in the performance also happens because of the defects introduced in the fabrication, such as open or short of vias and interconnects. Hence, the functional test of these embedded RF circuits becomes essential. Due to highfrequency measurement, functional test of integrated RF substrate (IRS) with ERPF is expensive because it requires two RF probes and expensive high-frequency equipments such as vector network analyzers (VNAs). Thus, to reduce the production cost of IRS, there is a high demand to test embedded RF circuits at low-cost.

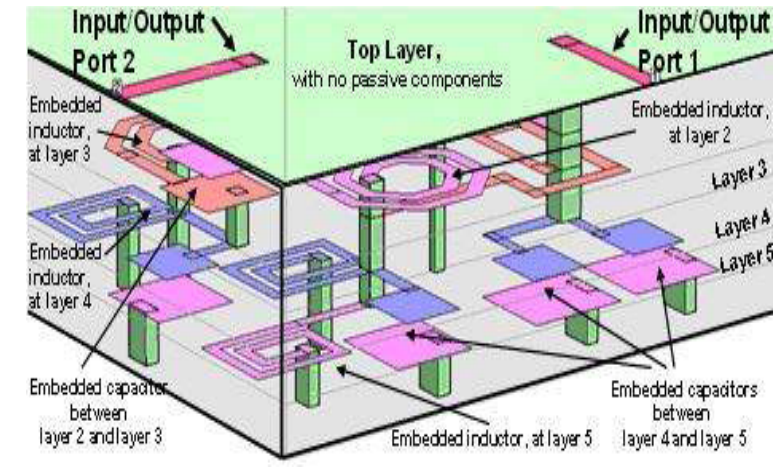

Figure 1. A model of integrated RF substrate with embedded filter

In the past, one-port test method based on resonator was proposed for embedded RF passives in [4], [5] and twoport test method based on pole and zero analysis was demonstrated to test RF filters in [6]. However, all of these methods require expensive instrumentation such as VNA. Low-cost test method without input test stimulus based on oscillation was proposed for analog and mixedsignal circuits in [7]. Method in [7] is based on reconfiguring circuit under test into an oscillator. As a result, it can not be applied for testing embedded passive filters, due to inaccessibility of internal nodes and absence of active component in ERPFs. The oscillation based test method for ERPFs was proposed in [8] in which an ERPF is included in the feedback network of an RF amplifier circuit such that this feedback causes the amplifier to oscillate. However, method in [8] requires measurement using two RF probes.

In this paper, a novel test method is proposed to test ERPFs by using a one-port measurement. We will be referring to this method as resonance-based testing in this paper. The proposed method reduces the test cost further as compared to method in [8], as it requires only one RF probe. In the proposed method, an ERPF is included in a RF oscillator circuitry by one-port probing, which causes a change in the oscillation frequency of the probe card because of the loading from ERPF. The test method is a low-cost solution because it reduces testsetup cost, as it a) needs only one RF probe, b) enables testing without VNA, and c) does not require any external test stimulus. 
To demonstrate this method, simulation results are shown for testing a $1.30-\mathrm{GHz}$ RF low-pass filter and a $1.50-\mathrm{GHz} \mathrm{RF}$ high-pass filter. As a proof of concept, measurements are shown to test $1.54-\mathrm{GHz}$ SMD lowpass passive filter. The proposed method is also implemented for testing $1.45-\mathrm{GHz}$ embedded RF lowpass filter.

The proposed test method is described in the following Section. In Section III, the test method is demonstrated with the help of modeling and testing of RF high-pass and low-pass filters. Measurements to test $1.54-\mathrm{GHz}$ low-pass filter are shown in Section IV In Section V, testing of embedded RF low-pass filter is demonstrated, which is followed by the conclusions and acknowledgments.

\section{Novel Proposed Test Method}

In the proposed Resonance-Based Test (RBT) method, a shift in the oscillation frequency of the test setup is used to detect failures in the Design Under Test (DUT). But it does not require reconfiguration or conversion of the DUT into an oscillator as is typically performed in conventional oscillation-based test methods. In the proposed method, the DUT is included in the existing oscillating system, so the presence of the DUT causes a shift in the oscillation frequency. The testing of the DUT is accomplished by careful analysis of this shift in the oscillation frequency, which is very sensitive to defects present in the DUT.

Consider the proposed test setup as shown in Fig. 2 in which an RF oscillator is connected to an embedded passive filter (DUT). The topology of this connection is established in such a way that the presence of the filter alters the oscillation frequency of the RF oscillator. The oscillation frequency of a RF oscillator changes because of the loading from an ERPF under test. This enables detection of parametric and catastrophic failures in the ERPF by monitoring the shift in the oscillation frequency of the test setup.

In this test method, the RF oscillator is designed in such a way that a) It can oscillate at the desired test frequency (Fo) after inclusion of the RF filter. Test frequency ( $\mathrm{Fo}$ ) can be chosen close to 3-db frequency of the RF filter b) The oscillation frequency of the RF oscillator changes from the good filter samples to the bad filter samples, and c) The change in the oscillation frequency $(\Delta \mathrm{f})$ can be measured using a low cost instruments.

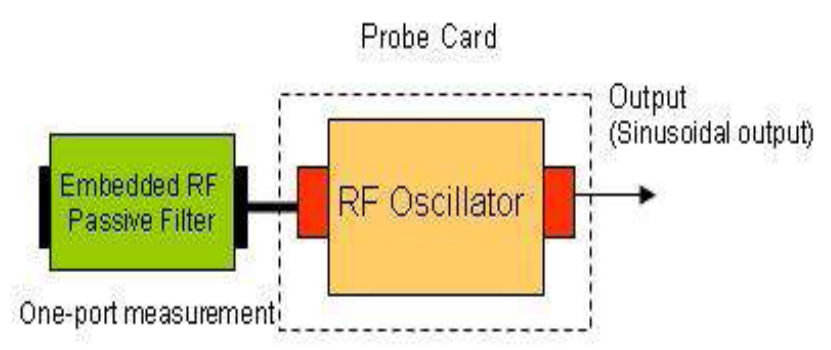

Figure 2 Proposed test setup of resonance-based test

By using few calibrating RF filter samples and known statistical distribution in the fabrication process of the embedded passives, estimation of the shift in the oscillation frequency $(\Delta f)$ is performed. Calibrating filters should be such that they represent the variations in the filter's performance due to the fabrication process. In simulations, statistical analysis like Monte Carlo [9] can be used.

In this test method, faults in embedded RF filters cause a shift in the oscillation frequency of the test setup around the response of the test setup with the golden filter. By the analysis of this shift in the oscillation frequency of the test setup, good and faulty RF filters are differentiated. If, for a particular fabrication technology of embedded RF filters, it becomes difficult to estimate the allowable shift in the oscillation frequency, then the output of the proposed test setup can be analyzed by a non-linear classification [10].

\section{Modeling AND Simulation}

In this section, the resonance-based test method is demonstrated by testing both RF low-pass filter (3-dB frequency of $1.30 \mathrm{GHz}$ ) and high-pass filter $(3-\mathrm{dB}$ frequency of $1.50 \mathrm{GHz}$ ). It is assumed that variations in the fabrication process of embedded filters across one sample are same, but it can be different between two samples. All simulations are done in Advance Design System (ADS). Same RF oscillator of type commonemitter negative resistance is used for these simulations.

\section{A. Low-pass Filter Modeling}

In this sub-section, RF low-pass filter (3-dB frequency of $1.30 \mathrm{GHz}$ ) is modeled and tested by the proposed test method. Seven samples of the filter are considered to demonstrate this test method. Insertion loss of these filters for the different values of inductance ( $\mathrm{La}$ ) and capacitance $(\mathrm{Ca})$ is shown in Fig. 3. It is assumed that 
known golden filter $(\mathrm{KGF})$ is $\mathrm{LP} 4(\mathrm{Ca}=2.4 \mathrm{pF}, \mathrm{La}=$ $12.4 \mathrm{nH})$, filters LP2, LP3, LP5, LP6 are marginally good filters, and $\mathrm{LP} 1(\mathrm{Ca}=1.80 \mathrm{pF}, \mathrm{La}=11.8 \mathrm{nH})$ and $\mathrm{LP7}$ $(\mathrm{Ca}=3.0 \mathrm{pF}, \mathrm{La}=13.2 \mathrm{nH})$ are bad low-pass filters.

Simulation setup as shown in Fig. 4 is used to test above RF passive circuits. When these RF low-pass filters were simulated with the RF oscillator, a corresponding change in the oscillation frequency of the RF oscillator was observed for different filters.

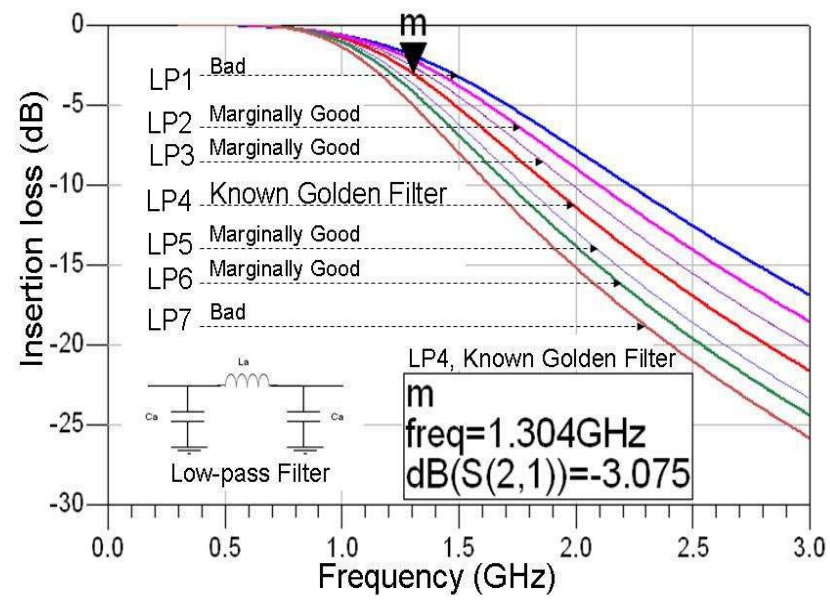

Figure 3 Insertion loss of RF low-pass filters under test

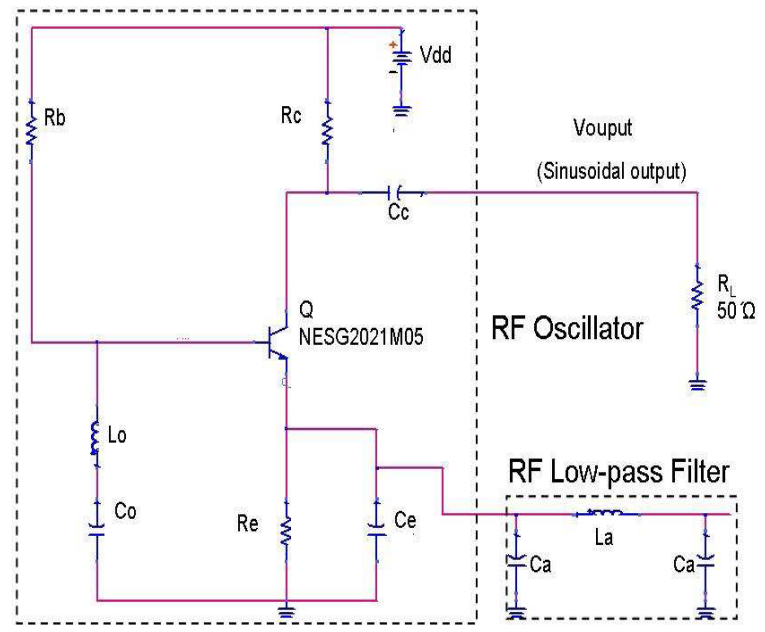

Figure 4 Simulation setup of the proposed resonance-based test method for RF low-pass filter testing

Based on the results shown in Table I, it can be inferred that the oscillation frequency of the test setup is around the response of KGF low-pass filter, LP4. Hence, testing is possible for these RF low-pass filters by defining allowable shift in the oscillation frequency around KGF's response. For example, for allowable shift of $\Delta \mathrm{f}_{1}+60$ $\mathrm{MHz}$ to $-20 \mathrm{MHz}$ around $1.23 \mathrm{GHz}$, filters LP1 and LP7 can be concluded as bad samples and LP2, LP3, LP5 and LP6 as marginally good RF low-pass filter samples.

\begin{tabular}{|c|c|c|c|}
\multicolumn{1}{|c}{ TABLE I. } & SIMULATION RESUTLS TO TEST RF LOW-PASS FILTERS \\
\hline RF Low-passfitter & $\begin{array}{c}\text { 3-db Frequeney } \\
\text { ofthe Filter }\end{array}$ & Filter's Classification & $\begin{array}{c}\text { Proposed Test-setup } \\
\text { Output } \\
\text { (RF oscillator) }\end{array}$ \\
\hline Sample LP1 & $1.46 \mathrm{GHz}$ & Bad & $1.33 \mathrm{GHz}$ \\
\hline Sample LP2 & $1.41 \mathrm{GHz}$ & Marginally Good & $1.29 \mathrm{GHz}$ \\
\hline Sample LP3 & $1.35 \mathrm{GHz}$ & Marginally Good & $1.27 \mathrm{GHz}$ \\
\hline Sample LP4 & $1.30 \mathrm{GHz}$ & $\begin{array}{c}\text { Known-golden-filter } \\
\text { (KGF) }\end{array}$ & $1.23 \mathrm{GHz}$ \\
\hline Sample LP5 & $1.24 \mathrm{GHz}$ & Marginally Good & $1.22 \mathrm{GHz}$ \\
\hline Sample LP6 & $1.21 \mathrm{GHz}$ & Marginally Good & $1.21 \mathrm{GHz}$ \\
\hline Sample LP7 & $1.15 \mathrm{GHz}$ & Bad & $1.19 \mathrm{GHz}$ \\
\hline
\end{tabular}

\section{B. High-pass Filter Modeling}

To further demonstrate this method, testing of a 1.50$\mathrm{GHz}$ RF high-pass filter is shown in this sub-section. In this example also seven samples of the RF filters are considered. It is assumed that the high-pass filter HP4 is $\mathrm{KGF}$ with $\mathrm{Ca}=1.0 \mathrm{pF}, \mathrm{La}=6.0 \mathrm{nH}$. Filters HP2, HP3, HP5 and HP6 are marginally good, and filters $\mathrm{HP} 1 \mathrm{Ca}=$ $0.75 \mathrm{pF}, \mathrm{La}=4.5 \mathrm{nH})$ and $\mathrm{HP7}(\mathrm{Ca}=1.25 \mathrm{pF}, \mathrm{La}=7.5$ $\mathrm{nH})$ are bad. A simulation test setup similar to Fig. 4 was used in this sub-section also. The summary of the simulation results is given in Table II.

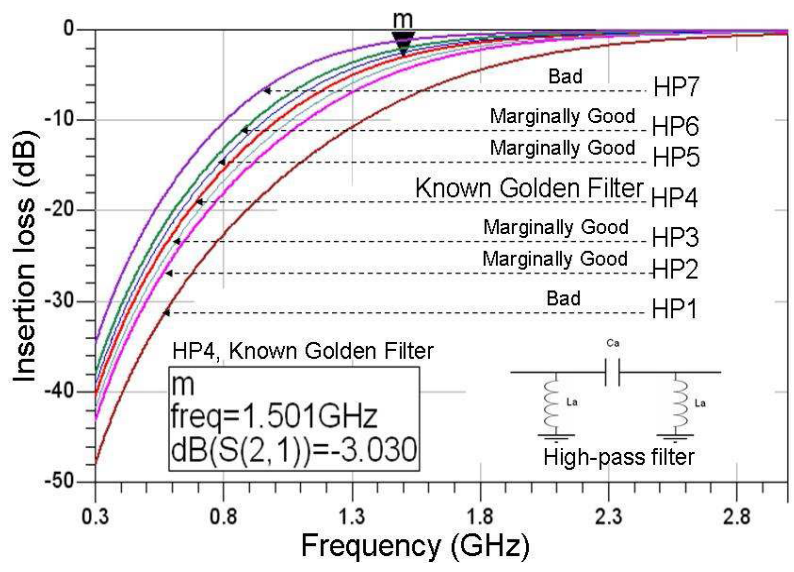

Figure 5 Insertion loss of RF hig-pass filters under test 
TABle II. Simulation Resutls to Test RF High-Pass Filters

\begin{tabular}{|c|c|c|c|}
\hline RF High-pass filter & $\begin{array}{c}\text { 3-db Frequency } \\
\text { of the Filter }\end{array}$ & Fitter's Classification & $\begin{array}{c}\text { Proposed Test-setup } \\
\text { Output } \\
\text { (RF oscillator) }\end{array}$ \\
\hline Sample HP1 & $2.00 \mathrm{GHz}$ & Bad & $1.25 \mathrm{GHz}$ \\
\hline Sample HP2 & $1.66 \mathrm{GHz}$ & Marginally Good & $1.23 \mathrm{GHz}$ \\
\hline Sample HP3 & $1.58 \mathrm{GHz}$ & Marginally Good & $1.17 \mathrm{GHz}$ \\
\hline Sample HP4 & $1.50 \mathrm{GHz}$ & $\begin{array}{c}\text { Known-golden-filter } \\
\text { (KGF) }\end{array}$ & $1.13 \mathrm{GHz}$ \\
\hline Sample HP5 & $1.43 \mathrm{GHz}$ & Marginally Good & $1.08 \mathrm{GHz}$ \\
\hline Sample HP6 & $1.36 \mathrm{GHz}$ & Marginally Good & $1.05 \mathrm{GHz}$ \\
\hline Sample HP7 & $1.20 \mathrm{GHz}$ & \multicolumn{3}{|c|}{ Bad } & $1.02 \mathrm{GHz}$ \\
\hline
\end{tabular}

In this example as well it is evident from Table II that the oscillation frequency of the simulation test setup is around the response of KGF high-pass filter, HP4. Therefore, for allowable oscillation shift $\left(\Delta \mathrm{f}_{1}\right)$ around the response of KGF, RF filter samples can be distinguished as bad and marginally good. For example, for $\Delta \mathrm{f}_{1}$ of $+100 \mathrm{MHz}$ to $-80 \mathrm{MHz}$ around $1.13 \mathrm{GHz}$, filters HP1 and HP7 can be concluded as bad samples, and HP2, HP3, HP5 and HP6 as marginally good samples.

\section{Measurements}

As a proof of concept, a low-pass filter (3-dB frequency $=1.54 \mathrm{GHz}$ ) was designed and assembled for the topology shown in the previous section by using different SMD capacitors and inductors. Among these filters, sample L2 is KGF (capacitor $=2.4 \mathrm{pF}$, inductor $=$ $8.2 \mathrm{nH}$ ) while sample L1 (capacitor $=2.0 \mathrm{pF}$, inductor $=$ $6.8 \mathrm{nH}$ ) and sample L3 (capacitor $=2.7 \mathrm{pF}$, inductor $=$ $8.7 \mathrm{nH}$ ) are its variants. To test these filters, a RF oscillator was also designed and assembled on the probe card. The insertion loss profile of the RF low-pass filters is shown in Fig. 6, and measurement results by the proposed test method are shown in Fig. 7 and summary of the obtained results is given in Table III.

Table III Summary of measurement results for testing RF low-pass filter by the proposed method.

\begin{tabular}{|c|c|c|}
\hline $\begin{array}{c}\text { RF low-pass } \\
\text { filter sample }\end{array}$ & $\begin{array}{c}\text { RF Low-pass } \\
\text { Filter's } \\
\text { 3-dB Frequency }\end{array}$ & $\begin{array}{c}\text { Proposed setup } \\
\text { output } \\
\text { (RF oscillator frequency } \\
\text { after inclusion of the } \\
\text { embedded RF filter) }\end{array}$ \\
\hline Sample L1 & $1.71 \mathrm{GHz}$ & $1.19 \mathrm{GHz}$ \\
\hline Sample L2 & $1.54 \mathrm{GHz}$ & $1.17 \mathrm{GHz}$ \\
\hline Sample L3 & $1.40 \mathrm{GHz}$ & $1.16 \mathrm{GHz}$ \\
\hline
\end{tabular}

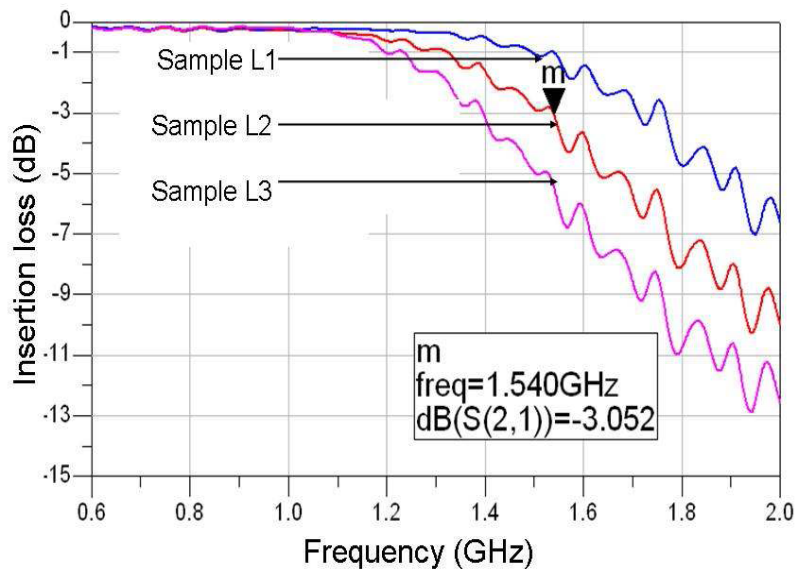

Figure 6 Insertion loss profile of the RF low-filter under test

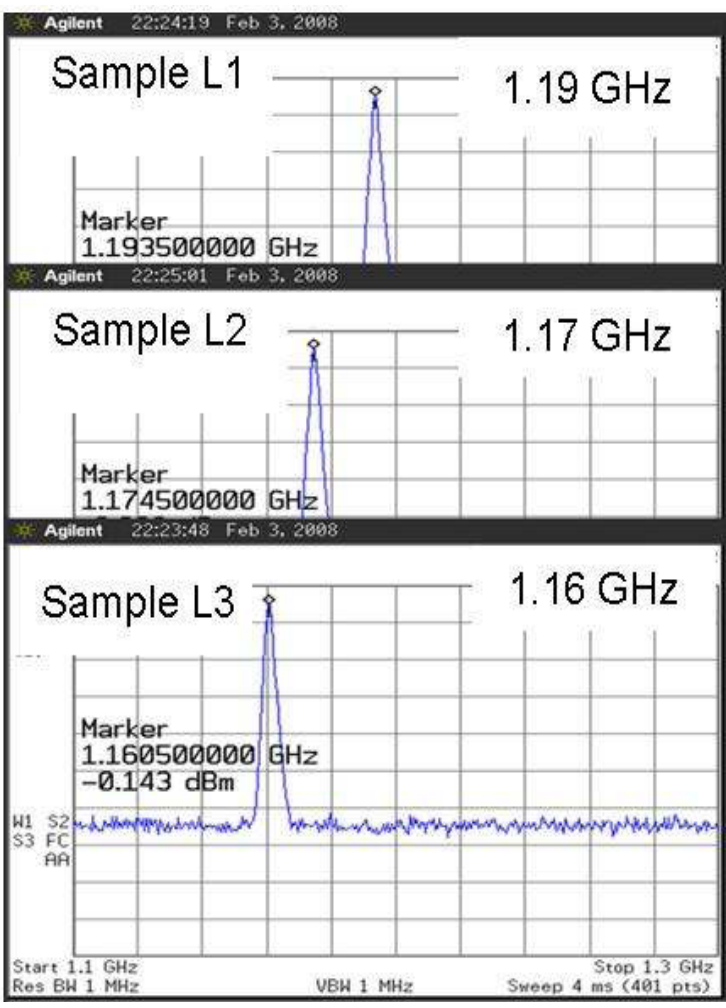

Figure 7 Measurement results for testing RF low-pass filter

It can be observed from the measurement results as shown in Table III that RF oscillator frequency changes for different RF filters. Hence, by comparing the shift in the oscillation frequency around the response of SampleL2 (KGF), testing of these filters is possible by the proposed method. 


\section{TESTING OF RF EMBEDDED FILTERS}

In this section, the proposed method is implemented for testing commercially-available embedded RF passive low-pass filter (3-dB frequency $\sim 1.45 \mathrm{GHz}$ ). The summary of the measurement results obtained using the proposed method are given in Table IV.

TABle IV. Summary of The Measurement Done to Test The EMBEDDED RF LOW-PASS FILTERS.

\begin{tabular}{|c|c|c|}
\hline $\begin{array}{l}\text { Samples of } \\
\text { RF Low-pass } \\
\text { Filter }\end{array}$ & $\begin{array}{l}\text { RF Low-pass } \\
\text { Filter's } \\
\text { 3-dB Frequency }\end{array}$ & $\begin{array}{l}\text { Proposed setup output } \\
\text { (RF oscillator frequency } \\
\text { after inclusion of the } \\
\text { embedded RF filter) }\end{array}$ \\
\hline A1 & $1.50 \mathrm{GHz}$ & $1.26 \mathrm{GHz}$ \\
\hline A2 & $1.49 \mathrm{GHz}$ & $1.26 \mathrm{GHz}$ \\
\hline A3 & $1.43 \mathrm{GHz}$ & $1.24 \mathrm{GHz}$ \\
\hline A4 & $1.44 \mathrm{GHz}$ & $1.24 \mathrm{GHz}$ \\
\hline A5 & $1.40 \mathrm{GHz}$ & $1.22 \mathrm{GHz}$ \\
\hline A6 & $1.40 \mathrm{GHz}$ & $1.22 \mathrm{GHz}$ \\
\hline
\end{tabular}

It can be noticed from Table. IV that the test setup has the same output oscillation frequency for the samples with same 3-dB frequency. Also, the test method gives different output oscillation frequency if $3-\mathrm{dB}$ frequency varies. For example, the output oscillation frequency for Samples A5 and A6 is around $1.22 \mathrm{MHz}$, while for Samples A1 and A2 the output oscillation frequency is around $1.26 \mathrm{GHz}$. Therefore, based on these measurement results it can be concluded that by this low-cost test method it is possible to get information about how good these embedded RF passive circuits are, by monitoring the shift in the output oscillation frequency of the test setup.

\section{CONCLUSION}

In this paper, a new test method called a resonancebased test is proposed to reduce test-setup cost for testing embedded RF passive filters (ERPFs). The test method is demonstrated by both simulations as well as measurements. Testing of a $1.30-\mathrm{GHz}$ low-pass filter and a $1.50-\mathrm{GHz}$ high-pass filter is demonstrated by simulations, while testing of a $1.54-\mathrm{GHz}$ low-pass filter is demonstrated by measurements. The method is also applied to the testing of an embedded 1.45-GHz RF lowpass filter.

Based on the simulation and the measurement results shown in this paper, it can be concluded that the proposed method can be used to test ERPFs by one-port measurement, and without any external test stimulus or vector network analyzer, causing a significant reduction in the test cost.

\section{ACKNOWLEDGMENT}

The authors would like to thank Prof. A. Chatterjee of the Georgia Institute of Technology, Atlanta, USA for his valuable discussions. The authors also like to thank JMD Inc, at Atlanta for providing samples of embedded filters.

\section{REFERENCES}

[1] Tummala, R.R.; Swaminathan, M.; Tentzeris, M.M.; Laskar, J.; Gee-Kung Chang; Sitaraman, S.; Keezer, D.; Guidotti, D.; Zhaoran Huang; Kyutae Lim; Lixi Wan; Bhattacharya, S.K.; Sundaram, V.; Fuhan Liu; Raj, P.M., "The SOP for miniaturized, mixed-signal computing, communication, and consumer systems of the next decade", IEEE Trans. Advanced Packaging, Volume 27, Issue 2, May 2004, pp. 250-267.

[2] Chang-Ho Lee; Sutono, A.; Sangwoo Han; Kyutae Lim; Pinel, S.; Tentzeris, E.M.; Laskar, J., "A compact LTCC-based Kuband transmitter module", IEEE Trans. Advanced Packaging, Volume 25, Issue 3, Aug. 2002 Page(s):374 - 384

[3] Kyutae Lim; Obatoyinbo, A.; Sutono, A.; Chakraborty, S.; Chang-Ho Lee; Gebara, E.; Raghavan, A.; Laskar, J., "A highly integrated transceiver module for $5.8 \mathrm{GHz}$ OFDM communication system using multi-layer packaging technology," Proc. IEEE International Microwave Symposium (IMS), May 2001, pp. 1739-1742.

[4] Kim, B.C.; Chatterjee, A.; Swaminathan, M.; Schimmel, D.E., "A novel low-cost approach to MCM interconnect test," Proc. IEEE International Test Conference (ITC), Oct 1995, pp. 184192

[5] Goyal, A.; Swaminathan, M.; Ward, C.; White. G; Chaterjee, A., "A Novel Method for Testing Integrated RF Substrate", Proc. IEEE Asia Pacific Microwave Conference (APMC), Dec 2007, pp. $277-280$.

[6] Heebyung Yoon; Junwei Hou; Chatterjee, A.; Swaminathan, M., "Fault detection and automated fault diagnosis for embedded integrated electrical passives", Proc. IEEE International Conference on Computer Design (ICCD), Oct. 1998, pp. 588 593

[7] Arabi, K.; Kaminska, B., "Testing analog and mixed-signal integrated circuits using oscillation-test method," IEEE Trans. Computer-Aided Design, Volume 16, Issue 7, July 1997, pp. 745-753.

[8] Goyal, A.; Swaminathan, M., "A Low Cost Method for Testing Integrated RF Substrates," Proc. IEEE International Microwave Symposium (IMS), June 2008, pp. (accepted).

[9] J.M. Hammersley and D.C. Handscomb, Morte Carlo Methods, New York: Chapman \& Hill, 1983.

[10] Stratigopoulos, H.-G.D.; Makris, Y., "Nonlinear Decision Boundaries for Testing Analog Circuits," IEEE Trans. Computer-Aided Design, Volume 24, Issue 11, Nov 2005, pp 1760-1773. 